\title{
Mittels App, online und vor Ort: Wie der BerlinCaseViewer die radiologische Fortbildung verändert
}

Lebenslanges Lernen ist mehr als nur ein Schlagwort in der Medizin. Jede Ärztin und jeder Arzt ist verpflichtet, sich auch nach dem Studium weiterzubilden. Inzwischen finden immer mehr Veranstaltungen online statt. Diesem Trend hat die CoronavirusPandemie zusätzlich Vorschub geleistet. Video-Konferenzen sind aber nur eine Möglichkeit für den Wissenserwerb im digitalen Zeitalter.

So schnell wie der Wissensschatz wächst, verändert sich auch die Lernkultur

"Па́vта ع“, dass sich alles in Bewegung befindet, wusste man bereits im antiken Griechenland. Goethe griff diesen Gedanken wieder auf, als er schrieb: „In demselben Flusse schwimmst du nicht zum zweiten Mal.“ Alle fünf Jahre, so die Faustregel, verdoppelt sich das medizinische Wissen. In die Wissensneuauflage, die sich längst nicht mehr drucken lässt, fließen neue Forschungserkenntnisse ein; überholte Praktiken verschwinden im Archiv. Relevante Studienergebnisse und neue Technologien kurbeln den medizinischen Fortschritt an. Im Bereich der Radiologie geben insbesondere technische Innovationen den Takt vor. Das betrifft neben der Patientenversorgung und Forschung auch die Lehre und Fortbildung. Die schiere Flut der Fortbildungsangebote kennzeichnet die eine Seite dieser Entwicklung, bedarfsorientierte Formate im Sinne von Personalized Learning die andere.

\section{Fortbildung ganz nach dem persönlichen Geschmack}

Das persönliche Lernerlebnis steht beim BerlinCaseViewer im Mittelpunkt. Frei nach dem Motto „Lernen, wann, wo und wie ich will“ gibt es die interaktive LernApp für Radiologie inzwischen für das iPhone, iPad und den Mac. Die umfassende Fallsammlung passt damit wahlweise in die Hosentasche; wer möchte, schaut sich die Spezialfälle im Detail auf dem großen Bildschirm an. Die Android-Version steht bereits in den Startlöchern und kommt im Laufe des Novembers. Damit macht der BerlinCaseViewer seine Fallsammlung mit den dazugehörigen Lern-Modulen noch mehr Menschen zugänglich.

Relevante Spezialfälle spannend aufbereitet

Mit Prof. Dr. med. Joachim Sieper, dem ehemaligen Leiter der Rheumatologie an der Berliner Charité, im Team hat sich der BerlinCaseViewer vor allem auf dem Gebiet rheumatischer Erkrankungen etabliert. Mehrere Module widmen sich den Ursachen chronischer Rückenschmerzen, darunter axiale Spondyloarthritis und Differenzialdiagnosen. Der interdisziplinäre Zugang kommt vor allem fachfremden Ärztinnen und Ärzten zugute. Denn die Fachleute hinter der App verstehen es, die Essenz jedes Thema herauszuarbeiten. Die Module führen anschaulich und interaktiv mithilfe von Quiz-Fragen und farblichen Overlays durch die Fälle, um das Wichtigste hervorzuheben. Wer das eigene Fachwissen testen möchte, beginnt am besten mit einem der kostenIosen Case-of-the-Month-Module. Neben muskuloskelettalen Erkrankungen des Bewegungs- und Stützapparates nimmt der BerlinCaseViewer indes auch das kardiopulmonale System in den Fokus. Dazu gehört beispielsweise das kostenlose Modul zur COVID-19-Pneumonie, das es bei den
2021 EuroMinnies Awards in der Kategorie „Best New Radiology Software“ zuerst auf die Shortlist und schließlich ins Finale geschafft hat.

\section{Internationales Fachwissen beim Berlin CaseViewer}

Eine wichtige Voraussetzung für den grenzüberschreitenden Erfolg der Software ist der mehrsprachige Auftritt. Namhafte internationale Expert*innen wie Dr. Fabio Macori (Italien), Dr. Yuranga Weerakkody (Australien) und Dr. Aline Serfaty (Brasilien) präsentieren ihre Fälle innerhalb des BerlinCaseViewers. Das Team ist überzeugt: Englisch erleichtert als Wissenschaftssprache den Austausch über Ländergrenzen hinweg; neues Wissen eignen sich Lernende aber besonders leicht in der eigenen Sprache an. Das zeigt sich auch daran, dass Social-Media-Lernkanäle aus aller Welt die Verbindung zur App nutzen, um ihre Fälle in größerer Tiefe darzustellen. Regelmäßig veranstaltet die BerlinFlame $\mathrm{GmbH}$, die hinter der App steht, auch Online-Live-Events und Kurse vor Ort. Ob auf dem kleinen oder großen Bildschirm, für iOS oder Android - der BerlinCaseViewer zeigt, wie vielseitig medizinische Fortbildung aussehen kann, um persönlichen Bedürfnissen gerecht zu werden. Am besten probieren Sie es selbst einmal aus.

Weitere Infos: www.berlincaseviewer.de Prof. Dr. Kay-Geert Hermann Charité - Universitätsmedizin Berlin 